\title{
Delayed presentation of deep penetrating trauma to the subaxial cervical spine
}

\author{
Julien Francisco Zaldivar-Jolissaint • \\ Lukas Bobinski • Yaelle Van Dommelen • \\ Marc Levivier · Christian Simon · John Michael Duff
}

Received: 8 August 2014/Revised: 10 November 2014/Accepted: 13 November 2014/Published online: 19 November 2014

(C) Springer-Verlag Berlin Heidelberg 2014

\begin{abstract}
Purpose To present a rare case of deep penetrating neck trauma in which a retained foreign body in the cervical spine (a broken knife blade) resulted in delayed radicular injury. We describe the surgical management using a retrojugular approach.

Case report Our patient sustained a stab wound to the supraclavicular triangle from a small pocketknife. He was initially managed in a local hospital by simple primary wound closure without any radiological examinations, and was discharged home. The patient re-consulted in a delayed fashion with mild local persistent neck pain. Subsequent radiological investigations revealed a foreign body (the broken blade of a pocket knife) embedded in the left neural foramen between the $\mathrm{C} 6$ and $\mathrm{C} 7$ vertebrae penetrating the disc space. The blade was lying between the left $\mathrm{C} 7$ nerve root and the ipsilateral vertebral artery (VA) at the transition of V1 and V2 segments. Initial neurological evaluation was normal. Some days later, the patient developed a delayed left C7 radicular deficit. We undertook urgent exploration along the wound corridor through a
\end{abstract}

J. F. Zaldivar-Jolissaint $(\bowtie) \cdot$ L. Bobinski · Y. Van Dommelen · M. Levivier - J. M. Duff

Service de Neurochirurgie, Centre Hospitalier Universitaire Vaudois (CHUV), University of Lausanne (Unil),

Rue du Bugnon 46, 1011 Lausanne, Switzerland

e-mail: julienzaldivar@gmail.com

C. Simon

Service d'ORL, Centre Hospitalier Universitaire Vaudois (CHUV), University of Lausanne (Unil), Lausanne, Switzerland retrojugular, transforaminal approach with successful removal of the blade.

Discussion To our knowledge, this is a unique case where a retained foreign body penetrated the soft tissues of the neck, embedding deep in the vertebral column without vascular, aerodigestive or significant primary neurological injury, while causing delayed neck pain and delayed onset radicular injury. We describe our surgical management for removal of the retained blade. The retrojugular approach gives excellent access to all of the important anatomical structures of the neck from an anterolateral approach.

Keywords Penetrating neck trauma - Foreign body · Delayed injury $\cdot$ Retrojugular approach - Cervical spine

\section{Introduction}

In treating penetrating neck injury, a rapid and systematic evaluation has been advocated [1,2]. Despite potentially devastating injury to vital structures, the entry wound can often be innocuous and thus easily overlooked or underestimated during the initial evaluation. Careful clinical and radiological diagnostic evaluations are necessary for planning potential surgical intervention, but also to avoid unnecessary surgical exploration [1, 3, 4]. For surgical exploration, the choice of approach depends on the localization of the injury as well as which structures require surgical repair. It may often require a multi-disciplinary surgical team.

We describe an unusual case of delayed presentation of deep cervical penetrating trauma in which a retained broken knife blade resulted in delayed radiculopathy. The details of surgical technique using a retrojugular approach are described. 


\section{Case report}

A 30-year-old male was involved in an altercation and sustained a stab wound to the left side of the neck. He presented at a local hospital in an intoxicated state. Physical examination was unremarkable. A small entry wound in the posterior triangle of the neck about $3 \mathrm{~cm}$ above the clavicle on the left side was sutured without radiological examination. The patient was discharged home. Sutures were removed a week later. On the 10th day after the assault, the patient had mild but increasing neck pain with movement, and returned to the hospital. The wound showed no signs of infection or inflammation (Fig. 1a). Standard X-rays of the cervical spine this time revealed a retained knife blade penetrating the $\mathrm{C} 6 / 7$ disc space, embedded in the left neural foramen between the C6 and C7 vertebrae (Fig. 1b). CT angiography (CTA) excluded a vertebral arterial injury (Fig. 2a-e). The blade was lying between the left $\mathrm{C} 7$ nerve root and the ipsilateral vertebral artery (VA) at the transition of V1 and V2 segments. The patient was transferred for further evaluation to our clinic.

On arrival, the cervical spine was immobilized with a Philadelphia collar. Neurological examination was normal. The laboratory investigations were unremarkable. Surgical exploration was recommended to the patient. The day before the planned surgery, the patient was found to have radicular pain and new onset left triceps weakness with diminution of the left triceps reflex, strongly suggesting a delayed left C7 radicular injury. Due to new onset neurological deficit, emergent surgery was performed.

\section{Surgical technique}

We undertook exploration along the wound corridor through a retrojugular, transforaminal approach. After intubation the patient was carefully positioned supine with the head placed on a horse-shoe holder, slightly turned to the right with neck extension. Neurophysiological monitoring was used throughout the surgery. An "L" shaped skin incision was made as shown (Fig. 3a). Subplatysmal flaps were raised anteriorly to the midline and posteriorly up to the trapezius. The sternocleidomastoid muscle was mobilized to expose the carotid sheath and its contents. Using a retrojugular approach as previously described [5-7], the longus colli muscles overlying the transverse processes and also lateral aspect of the body of the lower cervical vertebrae were exposed (Fig. 3b). The V1 segment of the ipsilateral vertebral artery (VA) was dissected out and controlled proximally with a vessel loop. The V2 segment of the VA was controlled distally between the foramen transversaria of C5 and C6 using a vessel loop, but was not transposed. The pharynx and larynx were mobilized anteriorly to expose the pre-vertebral cervical fascia. The blade was not visible but a lateral X-ray confirmed our level. Resection of a segment of the overlying longus colli muscles, and anterior retraction of the VA at the distal V1 segment enabled us to identify the broken off "stump" of the blade which was embedded in the C6/7 disc space (Fig. 3c). After dissection and identification of the extraforaminal $\mathrm{C} 7$ nerve root by direct stimulation, the blade was gently extracted from the $\mathrm{C} 6 / \mathrm{C} 7$ disc space across the $\mathrm{C} 7$ neural foramen (Fig. 3d). Posterior traction on the exiting nerve roots and the inferior trunk of the brachial plexus were unavoidable for "inline" removal of the blade along its axis of entry and to avoid "wanding" of the blade tip towards the thecal sac or towards the VA during extraction. Careful inspection of the intervertebral foramen did not reveal any CSF leak. However, inspection of the right $\mathrm{C} 7$ nerve root showed signs of minimal laceration of the epineurium and perineurium by the blade. These were sutured in two layers. After hemostasis, the wound was closed in multiple layers. Post-operatively the patient showed a mild C7 paresis, slightly worse than before surgery. Surgical manipulation of the already injured nerve root is the likeliest explanation for this. At 3 months followup, the triceps weakness had improved slightly.
Fig. 1 a Macroscopic appearance of the entry wound, 10 days after injury. b Cervical $\mathrm{X}$-ray showing the broken off knife blade embedded in the $\mathrm{C} 6 /$ 7 disc space
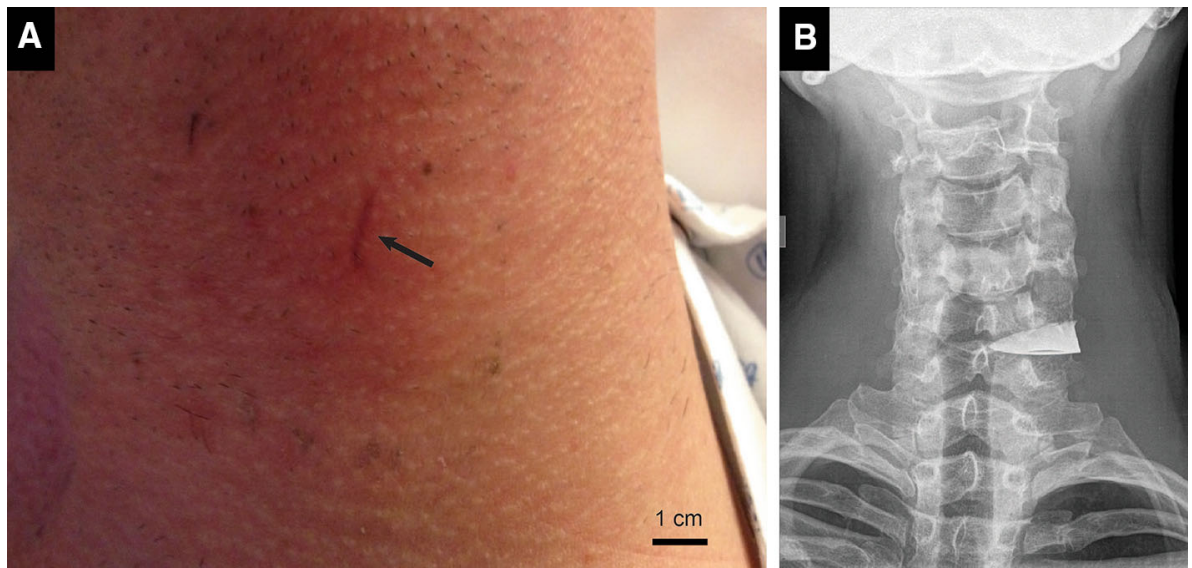
Fig. 2 a Coronal CT

angiography. b, c Sagittal CT angiography showing vertebral artery in relation to knife tip inside neuroforamen. $\mathbf{d}$,

e Transverse CT angiography through $\mathrm{C} 6$ and $\mathrm{C} 7$
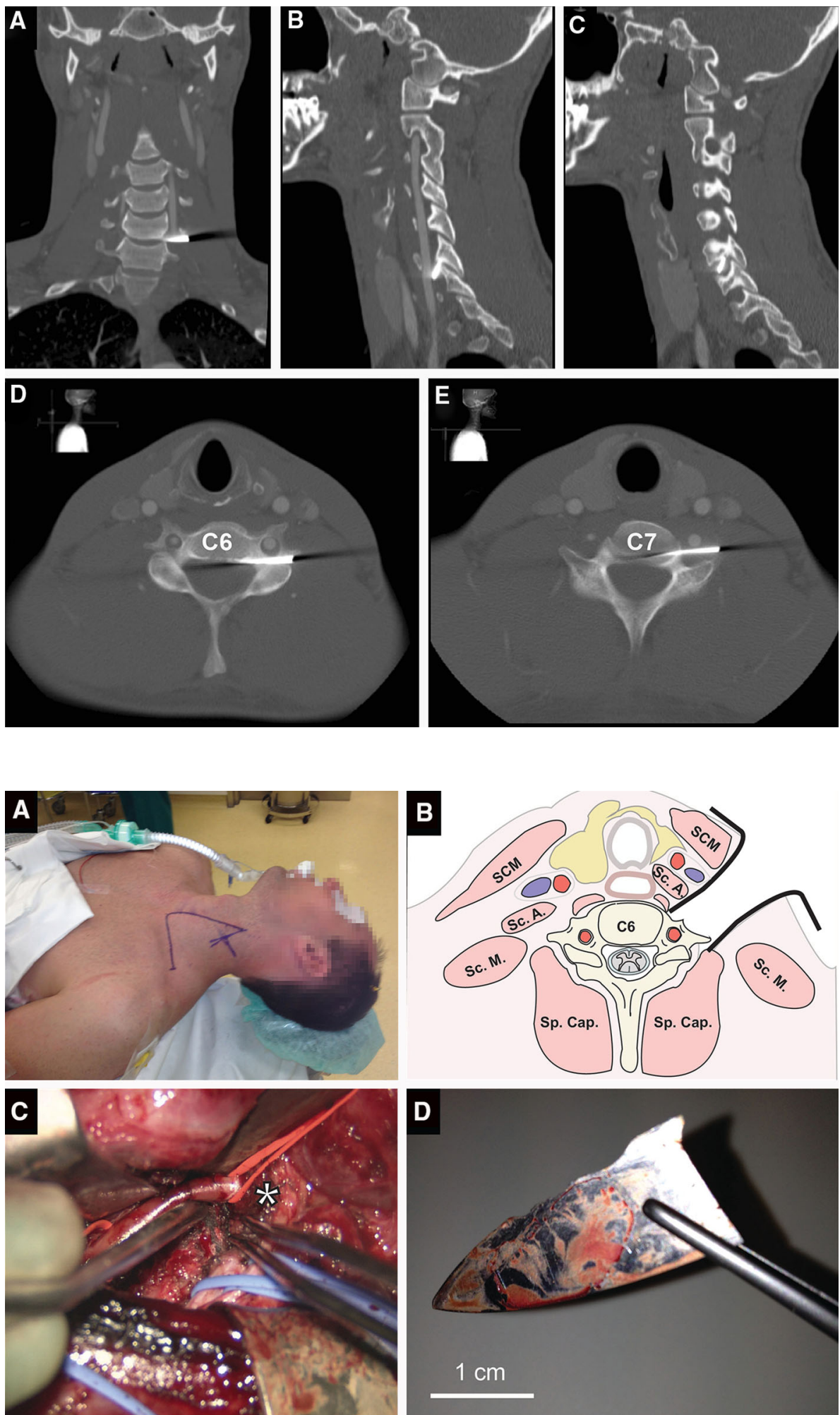

\section{Discussion}

Because of its high mortality and morbidity, penetrating neck injury requires emergent clinical and radiological evaluation to ascertain the integrity of the vital anatomical structures that are contained in the neck [1, 2]. Current management guidelines vow for guiding treatment through thorough physical examination and a low threshold for radiological examination. Mandatory surgical exploration has been abandoned in favor of a selective approach $[3,8]$. 
Any signs and symptoms of penetration injury to the respiratory tract, esophagus, vessels and/or neural structures should be investigated further and treated surgically when indicated. The approach is thus based primarily on clinical findings and a low threshold for CT angiography [3, 4, 9-12]. Further radiologic and endoscopic investigations are mandatory if there is suspicion of injury to the esophagus or trachea [13]. The goal is to avoid overlooking potentially life-threatening injuries, which might present in a delayed fashion.

There are reports about delayed neurological and vascular injuries in similar situations but these describe thoracic penetrating trauma and delayed medullary injury, or cervical trauma with immediate nervous, aerodigestive and/or vascular damage [14-17]. Our case is unique in that it involved the cervical spine, where the blade avoided the major blood vessels, airway, digestive tract, spinal cord and nerve trunks (except for a delayed minor laceration of the exiting left $\mathrm{C} 7$ nerve root) on its way to embed into the C6/ 7 disc space. It is also unique, as mechanical pain and 10 days delayed radicular deficit were potential indicators of blade micromovements and secondary injury. Coursing such a pathway of extraordinary anatomic brinksmanship, from skin to disc space, without serious injury to any of these structures seems a highly unlikely outcome. It challenges the surgeon to navigate around and between the same structures without causing further injury.

In cases of penetrating injury to the supraclavicular triangle, the retrojugular approach gives excellent access to all of the important anatomical structures of the neck from a lateral approach. Furthermore, it facilitates the early control of the VA and exploration of the neural foramina [6]. If necessary, this approach can easily be extended medially to have access to the anterior spine.

This case illustrates that the absence of clinical signs does not exclude serious injury in penetrating wounds of the neck, even with a delay in presentation, as in this case. Such wounds, even seemingly banal, require careful evaluation and appropriate investigations as described.

Conflict of interest The authors declare no financial conflict of interest.

\section{References}

1. Biffl WL, Moore EE, Rehse DH et al (1997) Selective management of penetrating neck trauma based on cervical level of injury. Am J Surg 174:678-682
2. Kendall JL, Anglin D, Demetriades D (1998) Penetrating neck trauma. Emerg Med Clin North Am 16:85-105. doi:10.1016/ S0733-8627(05)70350-3

3. Shiroff AM, Gale SC, Martin ND et al (2013) Penetrating neck trauma: a review of management strategies and discussion of the "No Zone" approach. Am Surg 79:23-29

4. Golueke PJ, Goldstein AS, Sclafani SJ et al (1984) Routine versus selective exploration of penetrating neck injuries: a randomized prospective study. J Trauma 24:1010-1014

5. Bruneau M, Cornelius JF, Marneffe V et al (2006) Anatomical variations of the V2 segment of the vertebral artery. Neurosurgery 59:ONS20-ONS24. doi:10.1227/01.NEU.0000219931. 64378.B5 (discussion ONS20-24)

6. Bruneau M, Cornelius JF, George B (2005) Anterolateral approach to the V2 segment of the vertebral artery. Neurosurgery 57:262-267 (discussion 262-267)

7. Lot G, George B (1997) Cervical neuromas with extradural components: surgical management in a series of 57 patients. Neurosurgery 41:813-820 (discussion 820-822)

8. Tisherman SA, Bokhari F, Collier B et al (2008) Clinical practice guideline: penetrating zone II neck trauma. J Trauma Inj Infect Crit Care 64:1392-1405. doi:10.1097/TA.0b013e3181692116

9. Bell RB, Osborn T, Dierks EJ et al (2007) Management of penetrating neck injuries: a new paradigm for civilian trauma. J Oral Maxillofac Surg Off J Am Assoc Oral Maxillofac Surg 65:691-705. doi:10.1016/j.joms.2006.04.044

10. Demetriades D, Theodorou D, Cornwell E et al (1997) Evaluation of penetrating injuries of the neck: prospective study of 223 patients. World J Surg 21:41-47 (discussion 47-48)

11. Gonzalez RP, Falimirski ME (1999) The utility of physical examination in proximity penetrating extremity trauma. Am Surg 65:784-789

12. Inaba K, Branco BC, Menaker J et al (2012) Evaluation of multidetector computed tomography for penetrating neck injury: a prospective multicenter study. J Trauma Acute Care Surg 72:576-583. doi:10.1097/TA.0b013e31824badf7 (discussion 583-584; quiz 803-804)

13. Sperry JL, Moore EE, Coimbra R et al (2013) Western Trauma Association critical decisions in trauma: penetrating neck trauma. J Trauma Acute Care Surg 75:936-940. doi:10.1097/TA. $0 \mathrm{~b} 013 \mathrm{e} 31829 \mathrm{e} 20 \mathrm{e} 3$

14. Fung CF, Ng TH (1992) Delayed myelopathy after a stab wound with a retained intraspinal foreign body: case report. J Trauma 32:539-541

15. Jones FD, Woosley RE (1981) Delayed myelopathy secondary to retained intraspinal metallic fragment. Case report. J Neurosurg 55:979-982. doi:10.3171/jns.1981.55.6.0979

16. Kulkarni AV, Bhandari M, Stiver S, Reddy K (2000) Delayed presentation of spinal stab wound: case report and review of the literature. J Emerg Med 18:209-213

17. Luo Y, Yuan H, Cao ZS (2013) Residual foreign body in the neck after trauma results in the delayed rupture of the common carotid and internal jugular vein: a case report. J Med Case Rep 7:13. doi:10.1186/1752-1947-7-13 